Como citar este artículo en APA: Alvarado Marambio, J. T. (2019).

Eucaristía, presencia real y viajes en el tiempo. Cuestiones Teológicas, 46 (106), 319-350. doi: http://doi.org/10.18566/cueteo.v46n106.a06

Fecha de recepción: 17 de septiembre de 2019 Fecha de aceptación: 14 de noviembre de 2019

\title{
EUCARISTÍA, PRESENCIA REAL Y VIAJES EN EL TIEMPO
}

\author{
Eucharist, Real Presence, and Time Travel \\ Eucaristia, presença real e viagens no tempo \\ José Tomás Alvarado Marambio
}

1 Doctor en Filosofía por la Universidad de Navarra, España. Profesor Asociado del Instituto de Filosofía, Pontificia Universidad Católica de Chile. Correo electrónico: jose.tomas.alvarado@gmail.com 


\section{Resumen}

El dogma de la transubstanciación definido por la Iglesia Católica genera varias cuestiones filosóficas. Estos problemas deben ser abordados desde una perspectiva más amplia que la usualmente adoptada en la discusión reciente en Teología filosófica analítica. Lo que ha sido enseñado es que la Eucaristía es un sacramento, pero al mismo tiempo es realmente un sacrificio. Y el sacrificio de la Misa es una "renovación" del sacrificio de Jesucristo en el Calvario. Se argumenta aquí que la mejor manera de comprender estas formulaciones es adoptando la tesis de identidad, esto es: el sacrificio de la Misa = el sacrificio de la cruz. La tesis de identidad tiene presupuestos metafísicos sustantivos que pueden haber parecido extravagantes a los padres del Concilio de Trento. Se argumenta aquí, sin embargo, que estos presupuestos son perfectamente coherentes.

Palabras clave: Eucaristía; Presencia real; Viaje en el tiempo; Transubstanciación; Espacio-tiempo.

\section{Abstract}

The dogma of transubstantiation defined by the Roman Catholic Church generates several philosophical questions. These problems should be addressed from a perspective broader than the one that has been usually adopted in the recent discussion in Analytic Philosophical Theology. What has been taught is that the Eucharist is a sacrament but at the same time a real sacrifice. And the sacrifice of the mass is a "renovation" of the sacrifice of Jesus Christ in the Calvary. It is argued here that the best way to understand these formulations is embracing the thesis of identity, $i$. e.: the sacrifice of the mass = the sacrifice of the cross. The thesis of identity has substantive metaphysical presuppositions that may have been seen as extravagant by the fathers of the Council of Trent. It is argued here, moreover, that those presuppositions are perfectly coherent.

Key Words: Eucharist; Real Presence; Time Travel; Transubstantiation; Space-Time. 


\section{Resumo}

O dogma da transubstanciação definido pela Igreja Católica gera várias questóes filosóficas. Esses problemas devem ser abordados de uma perspectiva mais ampla do que geralmente é adotada na discussão recente na Teologia Filosófica Analítica. O que foi ensinado é que a Eucaristia é um sacramento, mas ao mesmo tempo é realmente um sacrifício. E o sacrifício da missa é uma "renovação" do sacrifício de Jesus Cristo no Calvário. Argumenta-se aqui que a melhor maneira de entender essas formulaçóes é adotar a tese da identidade, ou seja: o sacrifício da massa $=$ o sacrifício da cruz. A tese da identidade tem pressupostos metafísicos substantivos que podem parecer extravagantes para os pais do Conselho de Trento. Argumenta-se aqui, no entanto, que esses orçamentos são perfeitamente consistentes.

Palavras Chave: Eucaristia; Presença Real; Viagem no Tempo; Transubstanciaçăo; Espaço-Tempo.

\section{Introducción}

La Eucaristía ha sido caracterizada al mismo tiempo como "sacrificio" y como "sacramento". En este trabajo se van a considerar algunas cuestiones de coherencia acerca de este misterio de fe, tal como es explicado por la Iglesia Católica². La Iglesia Católica ha enseñado de manera dogmática que en el sacramento de la Eucaristía se produce una transubstanciación del pan y el vino en el Cuerpo, la Sangre, el Alma y la Divinidad de Jesucristo ${ }^{3}$ que

2 Para una presentación de conjunto, cf. Catecismo de la Iglesia Católica, 1322-1419. Una excelente introducción desde una perspectiva histórico-crítica del sacramento de la Eucaristía y de sus raíces en la Antiguo Testamento en Pitre, 2011. Para presentaciones desde una perspectiva litúrgica, López, 2005; Fontbona, 2007.

3 Cf. Sínodo de Roma, confesión de fe de Berengario de Tours de 11 de febrero de 1079 (DH 700); carta Cum Marthae circa de Inocencio III al arzobispo Juan de 
se encuentran verdadera, real y sustancialmente presentes. Al declarar esto se ha hecho apelación explícitamente al concepto filosófico de substantia y no es extraño, por esto, que este misterio haya concentrado la atención de teólogos y filósofos durante siglos. En los últimos decenios se ha visto un renovado interés por problemas ontológicos. La eucaristía y las cuestiones que le son asociadas han vuelto a ser objeto de discusión en el marco de estos intereses generales (cf. Pruss, 2009, 2013; Pickup, 2015; Heil, 2015; Arcadi, 2016; desde otra perspectiva, Pickstock, 1998). En este trabajo se va a examinar una cuestión que no ha sido considerada expresamente en esta discusión reciente y que tiene que ver con la conexión existente entre el sacrificio del Calvario y el sacrificio de la Misa.

La posición explicada por el Magisterio de la Iglesia Católica ha sido caracterizada como una concepción en la que en la Eucaristía hay una "presencia real" de Jesucristo en las "especies" del pan y el vino y, al mismo tiempo, hay una "ausencia real" de la sustancia del pan y de la sustancia del vino (especialmente Pruss, 2009). Por un lado, esta posición contrasta con teorías que entienden la presencia de Jesucristo en la Eucaristía de un modo puramente "espiritual" o "simbólico". Contrasta también con teorías que entienden la presencia "real" de Jesucristo en conjunto con la presencia de la sustancia del pan y la sustancia del vino. Esto es, se ha sostenido que (Arcadi, 2016, p. 403): (i) el cuerpo, la sangre, el alma y la divinidad de Jesucristo están presentes verdadera, real y sustancialmente después de la consagración; (ii) ni el pan, ni el vino están sustancialmente presentes después

Lyon de 29 de noviembre de 1202 (DH 782); Definición contra los albigenses y los cátaros del Concilio IV de Letrán, 11 al 30 de noviembre de 1215 (DH 802); Profesión de fe del emperador Miguel Paleólogo del Concilio II de Lyon, Cuarta sesión de 6 de julio de 1274 (DH 860); Decreto para los jacobitas de 4 de febrero de 1442 del Concilio de Florencia (DH 1352); Decreto sobre el sacramento de la Eucaristía, 13a Sesión del Concilio de Trento de 11 de octubre de 1551 (DH 1642, 1652); Confesión tridentina de fe promulgada por la Bula Iniunctum nobis del papa Pío IV de 13 de noviembre de 1564 (DH 1866); Confesión de fe prescrita a los orientales, promulgada por la Constitución Nuper ad Nos de 16 de marzo de 1743 de Benedicto XIV (DH 2535); Condena de las doctrinas erróneas del Sínodo de Pistoya, dada por la Constitución Auctorem fidei de 28 de agosto de 1794 de Pío VI (DH 2629). 
de la consagración; y (iii) las cualidades sensibles o "especies" del pan y del vino siguen estando. Contra las teorías que sostienen que las palabras de la consagración indican solo una rememoración de lo sucedido en la Última Cena de Jesucristo con sus apóstoles, en (i) se enuncia que Jesucristo está verdadera, real y sustancialmente presente. Contra las teorías que sostienen que en la Eucaristía se encuentra sustancialmente Jesucristo y también se encuentra sustancialmente el pan y el vino ${ }^{4}$, se sostiene en (ii) que luego de las palabras de la consagración ya no están presentes ni el pan, ni el vino.

Hay una multitud de preguntas que estas formulaciones dogmáticas generan desde un punto de vista filosófico. Un tipo de cuestiones tienen que ver con la relación que deban tener las "especies" del pan y el vino con la sustancia del pan y la sustancia del vino. Si se trata de "accidentes", ¿cómo pueden subsistir sin la sustancia en la que ingieren? Se supone que un "accidente" es una entidad ontológicamente dependiente de una sustancia. Aquí pareciera requerirse la existencia de "accidentes" subsistentes, pues luego de la consagración, las especies del pan/vino deben poder existir sin la sustancia del pan/vino (Heil, 2015). Otro tipo de cuestiones tienen que ver con la localización de Jesucristo. ¿Cómo puede estar Jesucristo en el cielo, sentado a la diestra de Dios Padre y, al mismo tiempo, en cada hostia consagrada? ¿Cómo puede algo estar multi-locado en diferentes regiones del espacio "todo entero"? ¿Cómo puede suceder, además, que Jesucristo esté "todo entero" en cada parte de la hostia consagrada? (Pruss, 2009, 2013; Pickup, 2015). Todas estas cuestiones son de gran importancia y prometen clarificar-o, por lo menos, entender mejor-problemas ontológicos de un carácter general.

La transubstanciación, sin embargo, se produce en el contexto del sacramento de la Eucaristía del que se ha enseñado, tal como se indicó al principio, que es al mismo tiempo un auténtico sacrificio. La Eucaristía

4 Se ha denominado "consustanciación" la presencia conjunta de Jesucristo y de la sustancia del pan y de la sustancia del vino. Se ha denominado "empanación" la conexión entre el cuerpo de Jesucristo y la sustancia del pan y del vino como una "encarnación" de Jesucristo en el pan y el vino. Para detalles de estas posiciones heréticas, cf. Arcadi, 2016. 
es "renovación incruenta del sacrificio del Calvario" como ha explicado el Concilio de Trento (DH 1743). El Catecismo de la Iglesia Católica ha señalado que: "El sacrificio de Cristo y el sacrificio de la Eucaristía son, pues, un único sacrificio" (n. 1367). La clarificación de los problemas asociados a la transubstanciación no puede hacerse sin considerar con más detención la conexión que exista entre el sacrificio de la Misa y el sacrificio de la cruz. Tal como se verá, las formulaciones dogmáticas han visto en la transubstanciación un presupuesto precisamente para postular tal conexión. Lo que se va a mostrar aquí es que la forma más razonable de entender la conexión postulada en Trento es mediante la tesis de identidad:

Tesis de identidad: El sacrificio de la Misa = el sacrificio del Calvario

Esto es, que cada sacrificio de la Misa es numéricamente idéntico al sacrificio del Calvario, lo que implica que todas las Misas son un mismo y único evento. Esta tesis de identidad tiene presupuestos metafísicos muy sustantivos que debieron haber sido vistos con distancia por los padres conciliares de Trento. Lo que se explicará aquí, sin embargo, es que los motivos de sospecha que alguna vez pudieron tenerse respecto de tales presupuestos pueden ser contestados razonablemente.

En lo que sigue, por lo tanto, se van a explicar las formulaciones del Magisterio, especialmente en el Concilio de Trento, pero también en documentos posteriores de León XIII y Pío XII. Luego se va a considerar la coherencia de la tesis de identidad numérica entre la Eucaristía y el Calvario, para lo que se van a examinar sus presupuestos metafísicos -al menos, los que parecen más importantes- $\mathrm{y}$ cómo deba ser entendida la tesis de identidad. Lo que se explicará en este trabajo, por supuesto, es una reconstrucción racional del dogma de la Iglesia y no pretende ser más que una hipótesis adecuada que no obsta a otras interpretaciones diferentes.

\section{Renovación incruenta del sacrificio del Calvario}

Tal como se ha indicado, en el Concilio de Trento se ha sostenido que el sacrificio de la Eucaristía es la renovación incruenta del sacrificio del Calvario. 
En el capítulo 2 de la Doctrina sobre el sacrificio de la Misa se señala que en el sacrificio de la Misa:

se contiene e incruentamente se inmola aquel mismo Cristo (idem ille Christus continetur et incruente immolatur) que una sola vez se ofreció Él mismo cruentamente en el altar de la cruz.

Una sola y la misma es, en efecto, la víctima, y el que ahora se ofrece por el ministerio de los sacerdotes es el mismo que entonces se ofreció a sí mismo en la cruz, siendo solo distinta la manera de ofrecerse (sola offerendi ratione diversa). (22a Sesión de 17 de septiembre de 1562; DH 1743). (Los destacados son míos)

Lo que se indica en esta formulación es que hay identidad entre la víctima del Calvario y la víctima de la Misa, pero hay una diferencia en el "modo de ofrecerse", pues ahora se inmola de manera "incruenta"s. Para que pueda darse esta identidad de la víctima ofrecida es necesaria la transubstanciación, pues es por ella que Jesucristo está realmente bajo las "especies" del pan/vino. Por la transubstanciación llega a estar presente una sustancia que antes de la consagración no estaba presente "real y sustancialmente", mientras que un sacrificio es un evento. Lo que se está enunciando es que hay una peculiar relación entre el evento del Calvario y el evento de la Misa. La transubstanciación es un presupuesto de tal relación entre eventos, pero no puede ser identificado con ella.

5 El Concilio también señala en un par de ocasiones que la Misa es una repraesentatio del sacrificio de Calvario (DH 1644: quo mortis eius victoria et triumphus representatur; DH 1740: sacrificium quo cruentum illud semel in cruce peragendum representaretur). Esta expresión también había sido utilizada por Santo Tomás de Aquino para explicar el carácter sacrificial de la Misa (Hoc sacramentum dicitur sacrificium, in quantum repraesentat ipsam passionem Christi; Summa theologiae III, q. 73, a. 4, ad 3). Los especialistas han puesto de relieve, sin embargo, que en Trento no se utilizó "formalmente" esta noción de "representación" para caracterizar la relación entre el sacrificio de la Misa y la pasión de Cristo (cf. López, 2005, 113) y que no está claro si la expresión se utilizaba para designar el "hacer presente" de la pasión en la Misa o simplemente para designar una mera "representación teatral" (cf. Fontbona, 2007, p.169). 
De manera general, en efecto, un sacrificio es un tipo de evento. Es el ofrecimiento de algo en honor a la divinidad, para agradecerle los dones recibidos, para pedirle bienes y perdón por las ofensas. "Ofrecer" algo implica "separarlo" o "sacarlo" de sus usos ordinarios para ser dedicado a la divinidad. Algo "sagrado" o "sacro" es algo que ha sido objeto de esta "separación" o "segregación". En un sacrificio, la víctima es destruida para manifestar así la soberanía de la divinidad sobre todos los bienes que se poseen. En la Antigua Alianza están prescritos detalladamente diferentes sacrificios, para los que se especifican lugares, tiempos, utensilios y sacerdotes que ofician la ofrenda -que han de estar "separados" para YHWH y son, por ello, sagrados. En un evento de sacrificio, tal como se puede apreciar, intervienen una multitud de objetos: un sacerdote oferente, una víctima ofrecida -que puede ser un animal, o frutos de la tierra- utensilios sagrados cuyo uso está reservado para estos sacrificios, y construcciones en las que se debe desarrollar el sacrificio y que delimitan un espacio sagrado para ello. Pero un sacrificio no es simplemente la suma de todos estos objetos. Un sacrificio es un acontecimiento en que un sacerdote hace el ofrecimiento de la víctima, destruyéndola. Se trata, por lo tanto, de un "hacer" de alguien sobre algo en un lugar, y no simplemente ese alguien, ese algo y ese lugar. Es propio de un sacrificio un tiempo en el que la acción se desarrolla.

Hay diferencias importantes entre un evento y un objeto. Los "eventos" o "acontecimientos" son entidades tales como una fiesta, un partido de fútbol, una guerra, una batalla o una vida (Casati y Varzi, 2014, \$, p.1). Un evento posee esencialmente una localización espacio-temporal. En especial, un evento posee esencialmente una duración y partes temporales para cada parte de esa duración. Un partido de fútbol, por ejemplo, posee dos tiempos en los que se desarrolla y se puede concebir sin problemas como la suma de esas partes temporales. Un "objeto" o "sustancia", en cambio, es una entidad cuya persistencia en el tiempo no se da por el hecho de poseer ese objeto diferentes partes temporales para esos tiempos. Al menos, esto es lo que se ha comprendido tradicionalmente por un "objeto"6. No

6 Se han defendido en los últimos cincuenta años varias posiciones acerca de la persistencia de los objetos en el tiempo que contrastan con esta concepción tradicional. Algunos han sostenido que los objetos son, al igual que los eventos, 
parece tener sentido hablar, por ejemplo, de la "primera mitad de Sócrates". Sócrates no es una entidad con "partes temporales". En cambio, sí tiene sentido hablar de la "primera mitad de la vida de Sócrates". La vida de Sócrates, esto es, el conjunto de sucesos y vicisitudes de los que Sócrates ha sido parte, es, en cambio, un evento. En la concepción usual, un objeto persiste en el tiempo siendo idéntico en todos los instantes de su existencia o, si se prefiere, existiendo "todo entero" en cada instante de su existencia. Un objeto podría existir un tiempo mayor o menor del tiempo que, de hecho, existe. Si alguien muere a los 70 ańos, podría haber muerto a los 71 , o a los 69. Un evento, en cambio, no podría tener una duración mayor o menor de aquella que posee. No podría tampoco tener una localización espacio-temporal diferente de la que posee de hecho. Estas precisiones son importantes, porque cuando se sostiene que hay una transubstanciación del pan y del vino en el cuerpo, la sangre, el alma y la divinidad de Jesucristo, lo que se está sosteniendo es que hay un objeto bajo las especies del pan y del vino donde antes había otro objeto u otros objetos, a saber, el pan y el vino. Cuando se está sosteniendo, en cambio, que el sacrificio de la Misa es la renovación incruenta del sacrificio del Calvario, se está sosteniendo que el evento de la Misa es "renovación" del evento del Calvario.

Para comprender qué es lo que fue definido en Trento, es crucial entender de qué modo debe entenderse que un evento es "renovación" de otro y, en especial, de qué modo debe entenderse que la Misa es una "renovación" del sacrificio del Calvario. En abstracto, podrían describirse dos formas en que un evento puede ser la "renovación" de otro:

a. Una primera forma en que esto puede suceder es si hay un tipo general de evento que es reiterado en diversas ocasiones. Por ejemplo, una persona puede tener muchos cumpleaños durante su vida. Por supuesto, si se trata de un cumpleaños preciso, como cumplir cinco ańos, no puede ser que

entidades cuya persistencia está fundada en la posesión de partes temporales. Otros han sostenido que los objetos persisten en el tiempo por ser una secuencia de "contrapartidas temporales". Un objeto $x$ es una contrapartida de $y$ si es que (i) $x^{1}$ $y, \mathrm{y}$ (ii) $x$ es suficientemente semejante a $y$ en los respectos relevantes. No es posible explicar aquí estas diferencias. En lo que sigue en este trabajo, se va a suponer la concepción tradicional acerca de la persistencia de sustancias. 
alguien cumpla cinco años varias veces. Pero cada año se produce un cumpleaños, esto es, un evento que es una instancia del tipo de evento ser un cumpleaños. Lo que precisa el Concilio de Trento es que tanto en el sacrificio del Calvario como en cada sacrificio de la Misa hay una misma víctima, que es numéricamente idéntica en todos los casos: Jesucristo. Esto puede, en abstracto, interpretarse como un mismo tipo de evento que es reiterado muchas veces en diferentes tiempos y diferentes regiones del espacio. El tipo de evento de ofrecerse Cristo al Padre por inspiración del Espíritu Santo se realiza en todos esos casos, aunque esos eventos de sacrificio sean numéricamente diferentes entre sí. El Concilio de Trento impondría, de todos modos, una diferencia en el modo en que se produce el sacrificio (offerendi ratione diversa), pues en el Calvario es un sacrificio cruento, mientras que en una Misa no lo es.

b. Una segunda forma en que puede entenderse la "renovación" efectuada en el sacrificio de la Misa es como el hecho de que este es numéricamente idéntico al sacrificio de la cruz. No se trata simplemente de que vuelve a realizarse un mismo tipo de evento sino que se trata de exactamente el mismo evento. Esto es lo enunciado por la tesis de identidad enunciada arriba. Hay motivos importantes para adoptar la tesis de identidad, pues es una de las doctrinas centrales de la Epistola a los hebreos, en los capítulos 7 a 10, que el sacrificio de Cristo en la cruz fue único y definitivo para nuestra redención:

$<$ El sumo sacerdote, $s c$. Jesucristo $>$ no tiene necesidad de ofrecer sacrificios cada día, como aquellos sumos sacerdotes, primero por sus propios pecados, luego por los del pueblo. Y esto lo realizó de una vez para siempre ( $\tau$ oṽ $\tau$

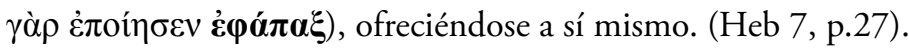

Mientras los sacerdotes del templo de Jerusalén deben ofrecer diariamente sacrificios imperfectos que no son suficientes para expiar por los pecados, Jesucristo ha expiado por todos los pecados de una sola vez y de manera definitiva al ofrecerse a sí mismo a Dios Padre por inspiración del Espíritu Santo. El problema que surge aquí es que si Jesucristo debe volver a ofrecerse y se ofrece en cada sacrificio de la Misa -aunque sea de manera "incruenta"- entonces pareciera que se niega el valor definitivo del sacrificio del Calvario. Los padres conciliares de Trento eran conscientes de 
este problema. Una de las acusaciones de los luteranos contra la santa Misa fue precisamente esta -fue hecha por un tal Urban Rieger en un escrito publicado en Augsburgo el 15297- y, por esto, se incluyó un canon de la Doctrina del sacrificio de la Misa respecto de este punto:

Canon 4. Si alguno dijere que por el sacrificio de la Misa se infiere una blasfemia al santísimo sacrificio de Cristo cumplido en la cruz, o que éste sufre menoscabo por aquél: sea anatema. (DH 1754).

Es obvio que si el sacrificio de la Misa y el sacrificio cruento de la cruz son numéricamente el mismo evento, no hay ninguna duda de que la Misa ni infiere blasfemia ni causa menoscabo al valor del Calvario. No puede ser, en efecto, que un mismo evento cause menoscabo a sí mismo8. Hay, entonces, muy buenas razones para preferir la tesis de identidad, de acuerdo con la cual la Misa es "renovación" del sacrificio del Calvario por ser idéntica al sacrificio del Calvario. Si es así, sin embargo, ¿por qué no se ha dicho expresamente en Trento? ¿Por qué expresar esta conexión con tantos circunloquios, tantas prevenciones acerca del carácter "incruento" de la Misa? Pareciera que los padres conciliares no quieren dar a entender que la Misa -en que está presente Jesucristo verdadera, real y sustancialmente por la transubstanciación- es un sacrificio en el que el Señor vuelve a sufrir una pasión. No quieren y no pueden dar a entender que el sacrificio del Calvario es, de algún modo, insuficiente y requiere reiterarse una y otra vez - tal cosa estaría en contradicción con la doctrina de la Epistola a los hebreos-. Sin embargo, tampoco se han atrevido a sostener expresamente que es el mismo evento. Parece haber razones de carácter metafísico por las que el magisterio de la Iglesia ha guardado una cuidada prudencia en estas formulaciones. En efecto, si cada sacrificio de la Misa es idéntico numéricamente al sacrificio del Calvario, entonces debemos sostener que

7 Esta crítica, por lo demás, está en coherencia con el rechazo de Lutero al carácter sacrificial de la Misa. Cf. Borobio, 2017, 410-411.

8 El Catecismo de la Iglesia Católica parece estar adoptando esta interpretación al señalar que: "El sacrificio de Cristo y el sacrificio de la Eucaristía son, pues, un único sacrificio." (n. 1367). El Catecismo señala esto precisamente al citar los textos del Concilio de Trento a que se ha hecho mención más arriba. 
hay una región espacio-temporal -la región espacio-temporal exacta en la que ocurre el sacrificio del Calvario- a la que tenemos acceso cada vez que asistimos a Misa, según se explicará con más detalle abajo. Estas son suposiciones que, naturalmente, deben haber parecido por lo menos dudosas a los padres conciliares en Trento.

Un examen del Magisterio ordinario posterior muestra que se ha mantenido esta cuidada prudencia en las formulaciones. Dos documentos son aquí dignos de mención. En la encíclica Caritatis studium de 25 de julio de 1898 del papa León XIII, dirigida a los obispos de Escocia se señala en la parte relevante que:

Aquella expiación [el sacrificio de la cruz] fue del todo perfecta y absoluta para los mortales; y de ningún modo hay otra, sino que la misma está incluida en el sacrificio eucarístico (ipsa illa in sacrificio eucharistico inest). Pero, así como sucedía que un rito sacrificial acompañaba la religión en todo momento, así el plan divinísimo del Redentor fue que el sacrificio, realizado una sola vez en la cruz (semel in Cruce consummatum), se convirtiera en perpetuo y perenne. Y la forma de esta perpetuidad consiste en la santísima Eucaristía, que no comporta solo una vana figura o un recuerdo de la realidad, sino la realidad misma, aunque de un modo distinto (sed veritatem ipsam, quamquam specie dissimili) (DH 3339).

Este documento se redactó para contestar la crítica de los protestantes al sacrificio de la Misa por tornar "insuficiente" el sacrificio de Cristo en el Calvario. Es elocuente que León XIII insista en que "no hay otro" sacrificio aparte del sacrificio de la cruz. ¿Cómo puede ser esto si es que se celebran regularmente Misas todos los días a menos que lo que sucede en la Misa sea lo mismo que sucedió en el Calvario? Estas consideraciones parecieran conducir naturalmente a admitir la tesis de identidad, pero León XIII parece detenerse justo en este punto. Todo lo que dice es que en la Misa "está incluido" o "se contiene" (inest) el sacrificio del Calvario, sin sostener abiertamente que la Misa es (est) el sacrificio del Calvario.

Otro documento en esta misma línea es la encíclica Mediator Dei de Pío XII de 20 de noviembre de 1947. Señala Pío XII: 
El sacrificio del altar no es una pura y simple conmemoración de la pasión y muerte de Jesucristo, sino que es un sacrificio propio y verdadero, por el que el Sumo Sacerdote, mediante su inmolación incruenta, repite lo que una vez hizo en la cruz (id agit, quod iam in cruce fecit) (...) (DH 3847).

Es diferente, en cambio, el modo como Cristo se ofrece (Dissimilis tamen ratio est, qua Christus offertur). En efecto, en la cruz El se ofreció a Dios totalmente y con todos sus sufrimientos, y esta inmolación de la víctima fue llevada a cabo por medio de una muerte cruenta, voluntariamente padecida; en cambio, sobre el altar, a causa del estado glorioso de su naturaleza humana, "la muerte no tendrá ya dominio sobre El" [Rom $6,9]$, y por eso la efusión de la sangre es imposible (...)

De este modo, la conmemoración de su muerte, que realmente sucedió en el Calvario, se repite (iteratur) en cada uno de los sacrificios del altar (...) (DH 3848).

Pío XII habla de "hacer lo que se hizo" (agere quod iam in cruce fecit) en el Calvario, lo que -nuevamente- puede ser entendido como la reiteración de un mismo tipo de evento o como el hacerse presente numéricamente el mismo evento. Se trata de la misma cautela que se ha visto en los padres conciliares de Trento.

\section{El problema metafísico}

Tal como se ha podido apreciar, es posible suponer que la motivación fundamental para las formulaciones de Trento y del Magisterio posterior tiene que ver con problemas metafísicos en los que los padres conciliares no han querido entrar. Esta actitud es perfectamente razonable. Por un lado, debe admitirse el carácter único y definitivo del sacrificio de Jesucristo en la cruz. Por otro lado, debe admitirse que Jesucristo está verdadera, real y sustancialmente presente en cada sacrificio de la Misa. Y debe admitirse que la Eucaristía es un verdadero sacrificio. La forma más natural de comprender conjuntamente estas proposiciones es adoptando la tesis de identidad. ¿’Pero cómo puede ser que algo que sucedió hacia el año 33 d.C. sea lo mismo que 
algo que sucede todos los días "desde que sale el sol hasta su ocaso" (Misal 1, p.11)? Si no se tiene una respuesta para este tipo de preguntas, lo prudente es dejar abierta la cuestión de si ha de identificarse el sacrificio de la cruz y la Misa. Lo que interesa ahora es, entonces, explorar con un poco más de detenimiento la tesis de identidad.

\subsection{Condiciones de identidad para un evento}

Para evaluar adecuadamente lo que se estaría sosteniendo con la tesis de identidad se hace necesario considerar las condiciones de identidad de un evento'. Si, en efecto, el sacrificio de la cruz es idéntico al sacrificio de la Misa, entonces tal identificación debería ser admisible de acuerdo con lo que tales condiciones de identidad estén especificando. Las condiciones de identidad para un tipo de entidad especifican las condiciones necesarias y suficientes para que dos entidades del tipo en cuestión -o lo que parecen ser dos entidades del tipo en cuestión- sean idénticas o diferentes entre sí. Respecto de los eventos se han propuesto una variedad de diferentes condiciones de identidad que pueden ser graduadas desde las menos exigentes a las más exigentes. De acuerdo con las condiciones de identidad menos exigentes puede suceder que dos eventos contarán como el mismo evento, mientras que deberían ser diferenciados de acuerdo con condiciones de identidad más exigentes. Quine, por ejemplo, sostuvo que un evento es una región del espaciotiempo (Quine, 1971, pp.63-65). Todo lo que podamos describir como acaeciendo en tal región son descripciones diferentes de lo que ontológicamente es el mismo evento. Kim ha sostenido, en cambio, que un evento es esencialmente la instanciación de una propiedad en un objeto en un tiempo o la instanciación de una relación en varios objetos en un tiempo. En la misma región del espacio-tiempo, por lo tanto, habrá tantos eventos como propiedades estén siendo instanciadas por el objeto o los objetos localizados en tal región (Kim, 1976). Supóngase que una esfera perfecta de color azul está rodando en el tiempo $t$. Para Quine, la región espacio-temporal ocupada por la esfera azul rodando es un único

9 Para una presentación general de las ontologías de eventos, cf. Casati y Varzi, 2014; Steward, 1997, pp.1-97. 
evento. Este único evento podría ser descrito como "hay una esfera rodando", como "hay una esfera" o como "hay algo azul". Todas estas serán descripciones de la misma entidad, tal como uno podría referirse a Sócrates como "el más sabio de los atenienses" o como "quien fue condenado por impiedad a beber la cicuta". Desde la perspectiva de Kim, en cambio, habrá que contar eventos diferentes si es que hay propiedades realmente diferentes que estén siendo instanciadas. La propiedad de tener forma esférica es diferente de la propiedad de ser de color azul, como también es diferente de la propiedad de rodar. Si esto es así, Kim contará tres eventos numéricamente diferentes entre sí donde Quine ha contado solo uno: el ser esférico de $x$ en $t$, el ser azul de $x$ en $t$ y el rodar de $x$ en $t$.

Se pueden dar diferentes descripciones lingüísticas de la misma propiedad. Por ejemplo, uno podría designar la propiedad de tener forma perfectamente esférica como "tener forma esférica", pero también como "tener la forma que Platón pensaba que tenía el cielo". Lo que resulta crucial para la especificación de las condiciones de identidad de Kim es qué propiedades estén siendo instanciadas, no qué descripciones de propiedades estén siendo dadas. De acuerdo con la concepción de Kim, son idénticos el evento de ser esférico $x$ en $t$ y el evento de tener $x$ en $t$ la forma que Platón creía que tenía el cielo, pues en ambos casos se está haciendo referencia a la misma propiedad. Una forma extrema de especificar las condiciones de identidad de un evento, sin embargo, sería sostener que los eventos son lo que enuncia una descripción o nominación de un objeto -u objetos- en un tiempo ${ }^{10}$. Desde esta perspectiva, los eventos están especificados tan finamente como las descripciones que sean utilizadas para expresarlos y tan finamente como el contenido cognitivo asociado a tales descripciones. Así, desde esta perspectiva, el evento de ser $x$ esférico en $t$ es diferente numéricamente del evento de tener $x$ en $t$ la forma que Platón creía que tenía el cielo. Alguien podría creer que existe el primer evento sin creer que existe el segundo, por ejemplo, porque podría no saber que Platón creía que el cielo tiene forma esférica. No puede suponerse, entonces, que "ser de

10 A veces se ha entendido la posición de Kim precisamente de este modo, debido a la manera en que se entiende una "propiedad". Ver la nota 8 abajo. 
forma esférica" y "tener la forma que Platón creía que tenía el cielo" poseen el mismo contenido cognitivo ${ }^{11}$.

Estas tres condiciones de identidad para eventos, entonces, pueden ser formuladas de este modo:

Condiciones de identidad - I Para eventos $e 1, e 2: e 1=e 2$ si y solo si la región espacio-temporal $r 1$ ocupada por $e 1=$ la región espacio-temporal $r 2$ ocupada por $e$.

Condiciones de identidad - II Para eventos $e 1, e 2: e 1=e 2$ si y solo si (i) $e 1$ es la instanciación de $P 1$ en $x 1$ en $t 1$, (ii) $e 2$ es la instanciación de $P 2$ en $x 2$ en $t 2$, y (iii) $P 1=P 2, x 1=x 2 \mathrm{y}$ $t 1=t 2$.

Las variables " $P 1$ ", " $P$ " tienen como rango propiedades. Las variables “ $x 1$ ", " $x 2$ " tienen como rango objetos. Las variables " $t 1$ ", " $t 2$ " tienen como rango tiempos, que pueden tener cualquier duración o ser instantes puntuales. Este principio de Condiciones de identidad - II puede ser generalizado para relaciones conectando $n$ objetos entre sí.

11 Un par de precisiones se requieren aquí acerca de qué sea una "propiedad". Del modo en que es entendido usualmente el concepto en metafísica analítica, designa una determinación, característica o cualidad de un objeto -o de varios objetos, si es que se trata de una relación- numéricamente diferente de él, que funda la existencia de semejanzas objetivas de ese objeto con otros, así como sus poderes causales. Qué propiedades existan -si son entendidas de este modo- es algo que normalmente se llega a saber por investigación empírica y no por mera reflexión a priori. Algunos, sin embargo, han entendido por "propiedad" lo que quiera que sea el valor semántico de un predicado de nuestros lenguajes. Si uno entiende las "propiedades" de este segundo modo, la concepción de Kim de los eventos se identifica con la tercera, pues habrá propiedades diferentes por cada descripción dotada de un cierto contenido cognitivo. Aquí, naturalmente, se asume la primera concepción. 
Condiciones de identidad-III Para objetos $e 1, e 2: e 1=e 2$ si y solo si (i) $e 1$ es el $D 1$ de $x 1$ en $t 1$, (ii) $e 2$ es el $D 2$ de $x 2$ en $t 2$, y (iii) $D 1=D 2, x 1=$ $x 2$ y $t 1=t 2$.

Las variables “ $D 1$ ”, “ $D 2$ ” tienen ahora como rango descripciones. Lo que se sigue de este principio de Condiciones de identidad-III es que dos eventos que son descritos de modo diferente son numéricamente diferentes entre $\mathbf{s i}^{12}$.

Si uno considera la tesis de identidad entre el sacrificio del Calvario y la Misa desde esta perspectiva, parece claro que el principio de Condiciones de identidad - III resulta inservible. La Misa es descrita de un modo diferente respecto del sacrificio de la cruz. Estas descripciones poseen un contenido cognitivo diferente. De hecho, no ha sido obvio ni siquiera para los padres conciliares de Trento si acaso estos eventos son o no el mismo evento. De acuerdo con el principio de Condiciones de identidad-III esto hace que, automáticamente, no sean el mismo evento. Pero este principio no es muy verosímil independientemente ${ }^{13}$. La cuestión, entonces, debe ser considerada desde la perspectiva que ofrecen los principios de Condiciones de identidad- I y II. De acuerdo al principio I, el evento del Calvario es idéntico al evento de la Misa si y solo si la región espacio-temporal en que ocurre el primer evento es idéntica a la región espacio-temporal en que ocurre el segundo.

12 En esta presentación se han omitido algunas posiciones en metafísica de eventos por parecen menos relevantes para la discusión que aquí se hace. Por ejemplo, Donald Davidson sostuvo que las condiciones de identidad de un evento están dadas por el conjunto de sus causas y de sus efectos (cf. Davidson, 1969). Por lo tanto, dos eventos que poseen exactamente las mismas causas y los mismos efectos son el mismo evento.

13 En efecto, de acuerdo con el principio de Condiciones de identidad-III los eventos son entidades "constituidas" por nuestros estados cognitivos. Dos eventos son diferentes si a algún sujeto aparecen como diferentes, pues si a alguien le aparecen como diferentes es que hay una descripción -un "modo de darse", un cierto contenido de información-que es aplicable a uno de ellos y no al otro. Se supone, sin embargo, que los eventos son relata de las conexiones causales y qué sea causa de qué no puede ser un artefacto de nuestras subjetividades. 
De acuerdo con el principio II, el evento del Calvario es idéntico al evento de la Misa si y solo si, ambos eventos están constituidos por exactamente los mismos objetos, las mismas propiedades y la misma ubicación temporal. Dado que un objeto ha de tener alguna localización espacial en un tiempo determinado, debe suponerse que también los dos eventos han de coincidir espacialmente.

Si se compara lo que exigen estos dos principios de Condiciones de identidad resulta que ambos coinciden en exigir la identidad de localización espacio-temporal para la identidad de dos eventos. En el caso del principio I esta identidad de localización es suficiente, además, para la identidad de los eventos. De acuerdo al principio II, en cambio, los eventos son algo más que una localización espacio-temporal. Son esencialmente la instanciación de una propiedad o una relación en un objeto o en varios objetos en un tiempo. En una misma localización espacio-temporal pueden darse varias instanciaciones de propiedades diferentes entre sí, lo que funda la existencia de diferentes eventos en tal locación ${ }^{14}$. Se ve que el principio II es más exigente que el I, pero ambos coinciden en requerir la identidad de localización en el espacio y el tiempo. Se ve también por qué la tesis de identidad resulta tan difícil de aceptar y por qué, también, los padres conciliares de Trento han buscado formulaciones tan cautelosas. La tesis de identidad requiere, por lo menos, la identificación de la localización espacio-temporal del sacrificio del Calvario y de cada sacrificio de la Misa. No solo el Calvario -el montículo con forma de calavera a la salida de Jerusalén- debe, de algún modo, ser el mismo lugar que cada Iglesia en la que se celebra la Misa, sino que también el tiempo en el que se crucificó a Jesucristo debe ser el mismo tiempo en el que se ha celebrado cada una de las Misas posteriores. Sin entrar en los requerimientos adicionales que impone el principio de Condiciones de identidad - II acerca

14 Por ejemplo, sea una esfera perfecta de color rojo rodando en una región espaciotemporal $r$. Si las propiedades de "ser de forma esférica", "ser de color rojo" y "rodar" son diferentes entre sí, la misma región alojará tres eventos numéricamente diferentes entre sí. También puede darse el caso de que la misma región aloje diferentes objetos. Por ejemplo, la región espacio-temporal $r$ puede estar cohabitada por una estatua y por el pedazo de bronce que la constituye. 
de la identidad de objetos y propiedades, no es extraño que suposiciones semejantes hayan sido tratadas con cautela.

\subsection{Viajes en el tiempo}

Ha sido tradicional concebir el tiempo como una secuencia de instantes ordenados por una relación de "ser posterior a" -o bien "ser anterior a". Ha sido también usual suponer que uno de esos instantes posee el carácter de "ser presente". Este carácter de "ser presente" se encuentra en "movimiento" desde los instantes pasados a los instantes futuros. La distinción entre pasado y futuro resulta por la partición que efectúa el presente. Todos los instantes anteriores al presente son pasados y todos los instantes posteriores al presente son futuros (Curtis y Robson, 2016, pp.65-87). Los eventos en esta perspectiva poseen una localización determinada en la serie temporal, lo que hace que tengan entre sí distancias temporales también determinadas. No pocos han sostenido, además, que solo existe el presente, esto es, los eventos o hechos presentes. Lo que denominamos "el pasado" es o bien el recuerdo, o bien alguna construcción abstracta que deba cumplir las funciones que normalmente le atribuimos a los hechos pasados. Lo que denominamos "el futuro" es o bien una expectativa o, nuevamente, alguna construcción abstracta apropiada (Curtis y Robson, 2016, pp. 89-113). Es obvio que, si solo existe el presente, entonces la tesis de identidad es sencillamente ininteligible. Ningún evento presente podría ser idéntico a un evento pasado porque no hay eventos pasados.

En el último siglo, sin embargo, ha ganado prevalencia una concepción del tiempo en la que no hay ningún privilegio ontológico para el presente -o lo que parezca ser el presente. En una concepción de este tipo los eventos pasados y futuros existen con tanto derecho como los presentes. Existe para nosotros una asimetría epistemológica entre los eventos pasados y los eventos futuros, pues podemos recibir información causal de los eventos pasados, pero no podemos -en principio- recibir información causal de los eventos futuros. Esto no hace que sean menos reales. La situación es análoga a lo que sucede con el espacio. Denominamos "aqui" al lugar donde se encuentra un hablante que está profiriendo esta expresión. Lo que quiera que sea "aquî" no es más ni menos real que otros lugares más o menos distantes. El lugar en que yo estoy no es ontológicamente excepcional. Del mismo 
modo, se debe denominar como "presente" a aquel instante en que está profiriendo esta expresión un hablante, pero sin que esto implique ningún carácter ontológicamente excepcional para el presente. Esta posición ha sido denominada "tetra-dimensionalismo", pues se trata al tiempo como una dimensión más junto con las tres dimensiones espaciales usuales. Se la ha llamado también "eternalismo", pues concibe la serie temporal completa como una realidad "ya dada" que puede ser "contemplada" como un todo. La serie temporal completa está "inmóvil". El movimiento consiste en el hecho de que un mismo objeto tenga propiedades incompatibles en diferentes tiempos de la serie o bien en el hecho de que en diferentes tiempos de la serie exista una diferente distribución de propiedades y relaciones. No hay, sin embargo, algo así como un movimiento de la serie temporal ${ }^{15}$.

Naturalmente, no es posible entra aquí a examinar las razones que han sido presentadas para adoptar una posición eternalista sobre el tiempo (Mellor, 1998; Sider, 2001, pp.11-52; Rea, 2003). No es difícil comprender, sin embargo, que una de las motivaciones centrales ha tenido que ver con el desarrollo de las teorías físicas de la relatividad especial y general. En la teoría de la relatividad especial la distención espacial y la distensión temporal de dos eventos es relativa a marcos de referencia inerciales. Sucede, sin embargo, que si uno contempla la distensión entre estos eventos en un espacio tetradimensional que incluye al tiempo como uno de sus componentes -el espacio-tiempo de Minkowski- entonces resulta que es posible precisar una distensión entre eventos que es invariante entre diferentes marcos de referencia. La teoría de la relatividad general amplía esta invariancia

15 Esto contrasta con posiciones que se han denominado "de Serie A" siguiendo el famoso trabajo de J. M. E. McTaggart (1908). La serie temporal se encuentra en permanente cambio, pues a cada instante el carácter de "presente" de alguno de los elementos de la serie está variando. Con la variación de qué instante sea presente, se produce también la variación en la distancia que todos los eventos pasados y todos los eventos futuros tengan con el presente. Los eventos futuros están "acercándose" al presente, mientras que los eventos pasados están "alejándose". Hay variaciones en este esquema si es que se sostiene que solo existe el presente, o que solo existe el presente y el pasado, pero no el futuro, o que solo existe el presente y el futuro, pero no el pasado. En cualquiera de estos casos, la serie temporal completa se encuentra cambiando permanentemente. 
para marcos de referencia acelerados. No es posible sacar consecuencias apresuradas acerca de qué deba seguirse de estas teorías para la ontología del espacio y el tiempo, aparte de su adecuación empírica (Dainton, 2001, pp.254-319), pero nos invitan a considerar el espacio-tiempo como una estructura geométrica unitaria -lo que obviamente es apropiado para una perspectiva eternalista del tiempo- $y$, además, como una estructura geométrica que posee una cierta topología. Hasta entrado el siglo XX había parecido obvio que "la" geometría del espacio es euclidiana y que esto es un hecho necesario y que puede ser descubierto a priori. Había parecido obvio también que el tiempo es una estructura unidimensional con un carácter intrínseco diferente del espacio. El surgimiento de las teorías de la relatividad especial y general, en cambio, ha llevado a pensar en la topología del espacio-tiempo como una cuestión empírica.

Desde estas perspectivas eternalistas ha llegado a tener sentido pensar en la coherencia de "viajes en el tiempo" que, de ser tema de ciencia ficción ha pasado a ser un tema legítimo para la reflexión filosófica. En los relatos típicos alguien utiliza un mecanismo para "ir" al pasado y luego para "regresar" al presente. El "viajero" se mueve en la dimensión temporal tal como lo hace en una dimensión espacial. Se han presentado varias objeciones de principio a la posibilidad de los viajes en el tiempo en este sentido. Estas objeciones tienen que ver con la posibilidad de loops causales, con la posibilidad de causalidad retroactiva y con el problema llamado "la paradoja del abuelo". No es posible hacer aquí una revisión ni siquiera somera de estas cuestiones, pero puede decirse con relativa seguridad que ninguno de estas objeciones de principio ha resultado decisiva (cf. Smith, 2013). Por supuesto, si solo existe el presente, un "viaje al pasado" no tiene ningún sentido. Lo que interesa considerar aquí, sin embargo, es la coherencia de un escenario semejante desde una perspectiva eternalista. Convendrá considerar un poco la "paradoja del abuelo" para mostrar por qué no hay aquí problemas de coherencia. Supóngase que Juan viaja al pasado con la intención de matar a su abuelo Diego. Juan compra un arma y le dispara. Juan depende causalmente de su abuelo. Si Juan mata a su abuelo, entonces hace desaparecer una de las causas de las que su misma existencia depende. Si no hay abuelo, no hay nieto. Entonces, si Juan viaja al pasado y mata a su abuelo, se "mata" a sí mismo. La acción de Juan de matar a su abuelo "borra" de la existencia al mismo Juan. Pero si no hay nieto, entonces tampoco hay nadie que mate 
al abuelo. Entonces, el abuelo puede engendrar al nieto. Sucede, por lo tanto, que la acción de Juan de matar a su abuelo tiene la insospechada consecuencia de que provoca la existencia de su abuelo y, con ello, su propia existencia. Todo esto parece absurdo. Un examen más detenido desde una perspectiva eternalista, sin embargo, muestra que no hay aquí incoherencia (cf. Lewis, 1976). Juan no mata a su abuelo. Juan depende causalmente de él. Si hay un "viaje en el tiempo", entonces no es que hay un pasado en que Diego engendra al padre de Juan, que engendra a Juan, el que luego es "modificado" por Juan retroactivamente. La serie temporal completa "ya fija" contiene los eventos que de hecho contiene -y podría contener otros, naturalmente- y en tal serie, por ejemplo, puede ser que Juan haya intentado matar a su abuelo. No hay un pasado que "transformar". Lo que se designa aquí como "pasado" intemporalmente contiene "siempre" los intentos de Juan. No hay contradicción, pues la proposición de que Juan mató a Diego es intemporalmente falsa, no es una proposición falsa que luego se hace verdadera por la acción de Juan.

La inteligibilidad de los viajes en el tiempo torna también inteligibles otros escenarios. Tal como se ha explicado más arriba, un objeto persiste en el tiempo existiendo "todo entero" en cada instante en que existe -o bien, siendo idéntico en cada instante en que existe-. Cuando se trata de la ocupación de una región del espacio, se supone que un objeto ocupa una región por poseer diferentes partes que, a su vez, ocupan sub-regiones de tal región espacial. Un objeto parece ocupar el espacio de un modo diferente del modo en que ocupa el espacio. Parece ocupar el tiempo existiendo "por entero" en cada lapso temporal. Parece ocupar el espacio, en cambio, por poseer partes propias que ocupan sub-regiones de una región dada. La coherencia de los viajes en el tiempo, sin embargo, hace que este contraste deba ser revisado. Supóngase que Juan el 1 de marzo del año 2016 a las 12:00 está en una cafetería. El año 2017 viaja al pasado al 1 de marzo de 2016 a las 12:00 y se sienta con él mismo en la cafetería. El año 2018 vuelve a viajar al 1 de marzo de 2016 a las 12:00 y también se sienta junto a sí mismo en la cafetería. Si uno contempla lo que sucede el 1 de marzo de 2016 a las 12:00 resulta que Juan está ocupando todo entero tres regiones del espacio desconectadas entre sí. Sea el espacio que ocupa Juan sentado en una silla de la cafetería el 1 de marzo de 2016 a las 12:00 la región $r$. Sea el espacio que ocupa Juan cuando viaje desde el futuro al sentarse ese mismo tiempo 
consigo mismo la región $r$. Sea, por último, el espacio ocupado por Juan cuando ha viajado desde el futuro una segunda vez al mismo tiempo la región $r$. Si Juan existe "todo entero" en cada instante de tiempo, entonces ha de existir todo entero al estar sentado en la cafetería el 1 de marzo de 2016 a las 12:00, al estar sentado ese mismo tiempo por segunda vez y al estar sentado ese mismo tiempo por tercera vez. No es que haya diferentes partes propias de Juan en cada una de las regiones $r, r$ y $r$. Juan está "todo entero" en cada una de esas regiones. La forma de estar extendido en el tiempo, entonces también se presenta en la ocupación del espacio. Juan es una entidad temporalmente extendida pero simple -pues no tiene partes temporales. Dada la inteligibilidad de los viajes en el tiempo, Juan también puede ser una entidad espacialmente extendida y simple -pues está "entera" en cada una de las regiones $r, r$ y $r$. No es extrańo, entonces, que se haya visto en este tipo de escenarios una forma de comprender cómo es que Jesucristo pueda estar multi-locado al producirse la transubstanciación (cf. especialmente, Pickup, 2015). Si una hipótesis de multi-locación es coherente para Juan también ha de serlo para Jesucristo presente en la Eucaristía.

\section{La tesis de identidad}

Uno podría estar inclinado a pensar que lo que ha sido propuesto para explicar la multi-locación de Jesucristo en la Eucaristía debido a la transubstanciación podría ser aplicado para comprender la tesis de identidad. Las diferencias entre un evento y una sustancia u objeto, sin embargo, obligan a tener aquí grandes precauciones. Para un objeto no es esencial cuál sea su localización espacio-temporal, con la sola excepción -tal vez- de su localización espacio-temporal en el primer instante de su existencia, si es que uno admite la necesidad de origen ${ }^{16}$. Un objeto localizado en la región espacial $r$ podría estar localizado en la región espacial diferente $r$. Un objeto

16 Se ha sostenido, en efecto, que las condiciones de origen de un objeto le son esenciales. Estas condiciones de origen incluyen, por supuesto, la región espacial exacta en que se ha comenzado a existir y el tiempo exacto en que se ha comenzado a existir. Cf. para una discusión general, Mackie, 2006, 47-78, 93-117. 
que existe entre el tiempo $t$ y el tiempo $t$, podría existir entre el tiempo $t$ y otro tiempo diferente $t$, anterior o posterior a $t$. Cuando se trata de un evento, en cambio, su locălización espacio-temporał le es esencial. Un evento no podría acaecer en un lugar espacial diferente de aquel en que ocurre de hecho, aunque la diferencia fuese infinitesimal. Un evento tampoco podría acaecer en un tiempo diferente del tiempo en que ocurre. No podría haber comenzado a producirse un poco antes de cuando ha ocurrido, ni podría haber terminado de ocurrir un poco después de cuando ocurre. Ni siquiera sería admisible una diferencia infinitesimal.

La cuestión, entonces, es cómo podría ser posible que la región espaciotemporal en que ocurre el sacrificio del Calvario sea idéntica a la región espacio-temporal en que se ofrece válidamente el sacrificio de la Misa. Lo que se requiere para algo así es que la topología del espacio-tiempo sea de tal naturaleza que la región del Calvario esté conectada de tal modo con las restantes regiones de la estructura espacio-temporal completa que sea accesible desde las regiones que circundan cada sacrificio de la Misa. Un escenario semejante puede parecer de entrada extraño, pero ya ha llegado a ser habitual pensar en la topología del espacio-tiempo con características que desafían el sentido común. Por ejemplo, piénsese en un mundo "plano" con solamente dos dimensiones. Los habitantes de este mundo podrían descubrir empíricamente que su mundo es finito, pero "sin bordes", si es que viajando en cualquier dirección un tiempo suficientemente largo llegan siempre al punto de partida. De un modo análogo, nuestro mundo podría tener una topología tal que, aún siendo finito, no tenga "bordes". Podría suceder que viajando un tiempo suficientemente largo llegásemos al mismo lugar y tiempo de partida. Si los viajes en el tiempo son metafísicamente imposibles tendríamos razones para rechazar la coherencia de una suposición semejante, pero ya se ha visto que no hay realmente razones suficientes para rechazar la posibilidad de viajes en el tiempo. Uno podría aquí aducir que la topología del espacio-tiempo que resultaría sería, tal vez, muy compleja. Pero qué puede importar esto a la omnipotencia divina. Es ciertamente mucho más sorprendente la Encarnación del Verbo en una naturaleza humana.

La tesis de identidad exige identificar el sacrificio del Calvario con cada sacrificio de la Misa y, con ello, identificar lugares y tiempos entre sí. ¿¿Se quiere sostener, entonces, que cada iglesia en la que se celebra una Misa es 
el Calvario en los momentos en que se está haciendo la celebración? Esto parece absurdo. Una iglesia es una construcción techada y el Calvario es un monte con forma de calavera. Durante la crucifixión de Jesucristo se estaban crucificando a dos personas también y había legionarios ejecutando la condena, miembros del Sanedrín, la madre del Señor, San Juan y María Magdalena. ¿Todos ellos también están en cada Iglesia mientras se celebra la Misa? Esto sería sorprendente, pues ninguna Iglesia tiene forma de monte y en las Misas no se ven otras personas crucificadas o legionarios romanos repartiéndose los despojos de los condenados. ¿ $\mathrm{O}$ es que se va a sostener que son solo las "especies" de la iglesia lo que nos resultan perceptibles mientras que "realmente" son otras las sustancias presentes? Pero nada de esto es necesario. El evento del sacrificio de Jesucristo puede ser localizado en el cuerpo de Cristo en la cruz y en el tiempo en que se ofrece a Dios Padre por inspiración del Espíritu Santo y tal ofrecimiento tiene su culminación en el instante preciso antes de su muerte. Esta es una región espaciotemporal mucho más acotada que todo Jerusalén el año $33 \mathrm{~d}$. C. Lo que se requiere para la tesis de identidad es que tengamos parte en el sacrificio de Jesucristo al morir en la cruz y podemos tenerlo si la región espaciotemporal de la Misa -la región exacta ocupada por las especies eucarísticas durante la Misa- se identifica con la región exacta del cuerpo de Jesucristo ofreciéndose en la cruz. Esto supone que el evento del Calvario tiene una incidencia causal mucho más ubicua que lo que uno pudiese suponer, pues resulta un evento contemporáneo a cada época en que se celebra el sacrificio de la Misa válidamente, pero esta es una consecuencia bienvenida y es lo que cabe esperar del evento que constituye la redención del mundo y la recapitulación de todas las cosas.

La tesis de identidad resulta coherente bajo el supuesto de una topología particular del espacio-tiempo. Para la comprensión de la transubstanciación, sin embargo, lo que ha sido propuesto es la multi-locación de Jesucristo (cf. Pruss, 2009, 526-533; Pickup, 2015). Esto es, el Señor está todo entero bajo las especies eucarísticas y, al mismo tiempo, en el cielo, "sentado a diestra de Dios Padre". ¿No podría adoptarse una posición de este tipo para comprender la identidad entre el sacrificio del Calvario y el sacrificio de la Misa? Así como en el ejemplo presentado arriba Juan está todo entero en cada una de sus tres locaciones paralelas, se podría -tal vez- pensar que el sacrificio del Calvario es un evento multi-locado todo entero en cada región 
del espacio-tiempo en la que se celebre la Misa válidamente. Esto haría innecesario postular una estructura topológica tan compleja para el espaciotiempo. Hay dos problemas, sin embargo, con esta hipótesis alternativa. En primer lugar, el modo en que existe un evento tanto en el tiempo como en el espacio es por la posesión de partes espaciales y partes temporales. Es incompatible con esta forma de ocupación del espacio-tiempo el llegar a ser una entidad simple espacio-temporalmente extendida. El caso presentado arriba de Juan multi-locado en tres regiones del espacio desconectadas entre sí se produce por el hecho de que Juan es una sustancia que persiste en el tiempo siendo idéntico en cada instante de su existencia. Cuando se trata de un evento, sin embargo, no persiste siendo idéntico en distintos instantes. Un evento no es una entidad simple temporalmente extendida. En segundo lugar, aún si se pudiese dar una respuesta aceptable al primer problema, dado que para un evento es esencial cuál sea su localización espacio-temporal, resultaría esencial para el sacrificio de la cruz estar también localizado en cada región espacio-temporal en que se celebra válidamente la Misa con posterioridad. Estaría inscrito en la misma naturaleza del sacrificio de la cruz que, por ejemplo, en el siglo XVI llegarían sacerdotes al valle central de Chile y se celebraría una Misa el 4 de junio de 1536. Como el sacrificio redentor de Jesucristo en el Calvario lleva inscrito en su misma esencia cada futura celebración de la Eucaristía, el sacrificio de la cruz haría necesaria casi toda la historia posterior de la Iglesia. Esto sería, naturalmente, excesivo. Presentaría también un problema para la libertad humana ${ }^{17}$, pues las celebraciones válidas de las Misas dependen -al menos en parte- de la decisión libre de personas humanas, como los sacerdotes celebrantes y los obispos bajo cuya autoridad se celebran. Lo razonable es suponer que es esencial al evento del Calvario su localización espacio-temporal, pero no es un hecho necesario cuál sea la topología del espacio-tiempo. El sacrificio del Calvario hubiese sido el mismo evento que es, aunque, por ejemplo, la primera Misa en Chile hubiese sido celebrada el 5 de junio de 1536 en vez del 4 de junio de 1536.

17 Suponiendo que la libertad -al menos, la libertad humana- es incompatible con el determinismo. Esto es, que si el sujeto racional $S$ decide libremente realizar la acción j, entonces $S$ pudo haber decidido no realizar j. 
Dicho esto, sin embargo, debe concederse multi-locación para el Señor con posterioridad al sacrificio de la Misa. El evento del Calvario es idéntico al evento de la Misa. Por la transubstanciación está presente en las especies eucarísticas Jesucristo "todo entero" durante este sacrificio -pues es el sacrificio del Calvario-, pero su existencia posterior al sacrificio no se produce porque, de algún modo, el sacrificio del Calvario siga durando mientras, por ejemplo, la Eucaristía sigue reservada en un sagrario para la adoración de los fieles. Después del único evento del sacrificio de la cruz el Señor se multi-localiza, persistiendo como una sustancia, existiendo "todo entero" en cada región espacio-temporal en la que se encuentre la Eucaristía. Toda Eucaristía es Jesucristo que se nos da directamente desde la cruz. Es su mismísimo cuerpo sacrificado en la cruz aquel en el que comulgamos. Por ejemplo, si se celebra una Misa en la Basílica de San Pedro en Roma el 8 de febrero de 2017, la región espacio-temporal ocupada por las especies eucarísticas entre la consagración y -tal vez- el ofrecimiento con el que concluye la Plegaria Eucarística ${ }^{18}$ es exactamente la misma región espaciotemporal del sacrificio del Calvario. El Señor está crucificado en un monte con forma de calavera a las afueras de Jerusalén. Una vez terminada la Plegaria Eucarística ${ }^{19}$, sin embargo, el Señor está en la Basílica de San Pedro, en Roma, el 8 de febrero de 2017, y está "todo entero" en cada hostia consagrada, multi-locado espacialmente ${ }^{20}$.

18 Es discutible cuál sea exactamente el lapso en el que se produce la identificación del sacrificio del Calvario y el sacrificio de la Misa. Por lo menos debe incluir el instante de la consagración, cuando el sacerdote dice válidamente, de acuerdo con el Canon Romano: Accipite et manducate ex hoc omnes: hoc est enim corpus meum, quod pro vobis tradetur y luego Accipite et bibite ex eo omnes: hic est enim calix sanguinis mei novi et aeterni testamenti, qui pro vobis et pro multis effundetur in remissionem peccatorum. Hoc facite in meam commemorationem. No es claro, sin embargo, si el evento se extiende durante todo el ofrecimiento sacrificial que se hace en la Plegaria Eucarística y que concluye con: Per ipsum, et cum ipso, et in ipso, est tibi Deo Patri omnipotenti, in unitate Spiritus Sancti, omnis honor et gloria per omnia saecula saeculorum.

19 O quizás antes, de acuerdo con lo explicado en la nota 15 anterior.

20 Se puede apreciar, por esto, que el cuerpo de Cristo presente realmente en la Eucaristía no es su "cuerpo glorioso" si esta hipótesis es correcta. Por lo menos, no puede ser el "cuerpo glorioso" de Cristo en el momento de la consagración por ser este evento idéntico al sacrificio del Calvario. Esto no obstaría para que la Eucaristía reservada para la adoración de los fieles con posterioridad al sacrificio de la Misa 
Se puede apreciar también que la tesis de identidad es una razón para rechazar la posición de que Jesucristo está presente en la Eucaristía de un modo "sacramental", que no es el modo en que un objeto se encuentra en un lugar (cf. Santo Tomás de Aquino, Summa theologiae, III, q. 75, a. 1, ad 3). La tesis de identidad exige que Jesucristo en encuentre localmente en la región espacio-temporal en que se celebra el sacrificio de la Misa. Se trata, en efecto, de la misma región espacio-temporal en que se produce el sacrificio de la cruz, y Jesucristo está ciertamente presente de manera local en el Calvario cuando ha sido crucificado. Por lo tanto, el Señor no puede estar de un modo no local en el sacrificio de la Misa. No es razonable suponer que luego de estar presente de manera local en el sacrificio de la Misa, Jesucristo pase a estar solo "sacramentalmente" presente en las especies consagradas después. Es entendible que se postule esta presencia "sacramental" si es que no hay claridad acerca de la coherencia de la multi-locación de un objeto, pero existiendo ahora tal claridad, lo más verosímil es suponer que la presencia local del Señor en el sacrificio de la Misa continúa luego en la presencia local del Señor en la región ocupada por las especies eucarísticas. Se ańade a este problema el que sea difícil hacer sentido de qué sea una "presencia sacramental", sin entenderla simplemente como la presencia de Dios en todo espacio y todo tiempo (Pruss, 2009, pp.518-523) ${ }^{21}$. Si la presencia de Jesucristo en la Eucaristía es la misma omnipresencia de Dios en toda su creación, no hay nada especial en la Eucaristía aparte de ser, tal vez, un símbolo.

\section{Conclusiones}

sea el cuerpo de Cristo en estado glorioso.

21 Un sacramento es un signo sensible y eficaz de la gracia. En principio, una presencia "sacramental", no local, debería ser un modo de estar presente Jesucristo por algún signo que sea, al mismo tiempo eficaz de la gracia de Cristo. Si esto no se entiende como una presencia local, no parece ser algo diferente de la forma en que Dios está presente "por potencia" en toda la creación, por estar toda la creación bajo la eficacia de su acción. Lo mismo podría decirse de la doctrina luterana acerca de la presencia illocaliter del Señor en la Eucaristía. 
Se ha presentado en este trabajo la cuestión acerca de cómo el sacrificio de la Misa sea "renovación incruenta" del sacrificio del Calvario, siguiendo las formulaciones del Concilio de Trento sobre la Eucaristía. La forma más razonable de entender esta conexión entre cada Misa y el sacrificio de la cruz es suponiendo que se trata de eventos numéricamente idénticos. Los padres conciliares, sin embargo, han escogido formulaciones mucho más cautelosas. Había buenos motivos para hacerlo, pues la tesis de identidad entre el sacrificio del Calvario y el sacrificio de la Misa tiene presupuestos metafísicos muy sustantivos que en el siglo XVI deben haber parecido, por lo menos, sospechosos. Esta cautela se ha mantenido en el magisterio ordinario posterior.

Se ha mostrado aquí, sin embargo, que esos presupuestos son perfectamente aceptables, si es que se admiten algunas posiciones metafísicas defendidas hoy de manera bastante general -aunque discutidas, por supuesto. Las condiciones de identidad de eventos requieren la identificación de la región espacio-temporal ocupada por estos. Para que esta identificación tenga sentido se requiere postular una metafísica eternalista del tiempo en la que todos los eventos pasados, presentes y futuros poseen el mismo estatus ontológico. Se requiere también que la topología del espacio-tiempo permita que regiones "pasadas" sean accesibles desde regiones "futuras". Este tipo de escenarios son los que se han contemplado en los "viajes en el tiempo", cuya coherencia ha sido examinada con detención en la discusión filosófica de los últimos cincuenta años. Sin necesidad de defender directamente estas posiciones metafísicas sustantivas, se puede ver que los presupuestos de la tesis de identidad que en el siglo XVI pueden haber parecido extravagantes resultan ahora perfectamente inteligibles.

La tesis de identidad, también, permite comprender mejor la transubstanciación y algunos de los problemas que trae consigo. Se ha propuesto recientemente que podría entenderse como un caso de multilocación de una sustancia que ha de ser concebida como una entidad "simple extensa" (Pickup, 2015), pero también se ha propuesto que podría explicarse por una "curvatura" del espacio-tiempo (Pruss, 2009). La tesis de identidad, sin embargo, exige una topología especial o "curvatura" del espacio-tiempo. Pero la persistencia de Nuestro Señor en las especies eucarísticas con 
posterioridad al sacrificio de la Misa, en cambio, exige postular su multilocación espacio-temporal como una entidad "simple extensa".

Se puede apreciar en este examen, además, un ejemplo muy concreto acerca de cómo el "diálogo" entre lo que muestran los datos revelados y lo que puede llegar a conocerse por el mero uso de la razón, sin apelación a lo que se conoce por fe sobrenatural, resulta fructífero tanto para una mejor comprensión de los misterios de fe, como para una mejor comprensión del mundo. La tesis de identidad es, por sí misma, mucho más verosímil que sus alternativas desde una perspectiva teológica. Esto es un motivo muy fuerte para admitir sus presupuestos metafísicos -al menos, lo es para mí. En sentido contrario, la verosimilitud independiente de estas posiciones metafísicas acerca del eternalismo en metafísica del tiempo, la posibilidad de "viajes" en el continuo espacio-temporal y la ontología de eventos confirma la plausibilidad de la hipótesis teológica ${ }^{22}$.

\section{Referencias}

Aland, B., Aland, K., Karavidopoulos, J., Carlo, M. Marini y Bruce, M. (2012). Nestle-Aland Novum Testamentum Graece. $28^{a}$ Edición revisada. Stuttgart: Deutsche Bibelgesellschaft

Ubieta López, J.A (ed.). (2009). Biblia de Jerusalén, Bilbao, Espańa: Desclée de Brouwer Juan Pablo II. (1992). Catecismo de la Iglesia Católica

Arcadi, J.M. (2016). Recent Philosophical Work on the Doctrine of the Eucharist, Philosophy Compass, 11(7), pp.402-412

Borobio, B. (2017). Lutero y la Eucaristía en su contexto, Phase 57, pp.405-434

22 Las ideas de este trabajo han tenido una larga gestación a través de los años. Debo agradecer las conversaciones sobre este tema con Alexander Pruss, Martin Pickup y Miguel González. Agradezco también las sugerencias de un par de evaluadores anónimos de esta revista. 
Casati, R. y Varzi, B. (2014). Events. Stanford Encyclopedia of Philosophy. Disponible en: https://plato.stanford.edu/entries/events/. Consultado el 3 de febrero de 2017

Curtis, B y Robson, J. (2016). A Critical Introduction to the Metaphysics of Time. Londres, Inglaterra: Bloomsbury Academic

Dainton, B. (2001).Time and Space. Chesham, Inglaterra: Acumen

Davidson, D. (2001). The Individuation of Events, en Essays on Actions and Events. Oxford, Inglaterra: Clarendon Press, pp.161-180

Denzinger, H. y Hünermann, P. (eds.). (1999). Enchiridion symbolorum definitionum et declarationum de rebus fidei et morum. Barcelona, España: Herder

Fontbona, J. (2007), La cena del Señor, misterio de comunión, Barcelona, España: Centre de Pastoral Litúrgica.

Heil, J. (2015). Cartesian Transubstantiation, Oxford Studies in Philosophy of Religion, 6, Oxford: Oxford University Press, pp.139-157.

Kim, J. (1976). Events as Property Exemplifications en Myles Brand y Douglas Walton (eds.), Action Theory, Dordrecht: Reidel, pp. 159-177

Lewis, D. (1976), The Paradoxes of Time Travel, American Philosophical Quarterly 13 , pp. $145-152$

López, J. (2005). La celebración eucarística, centro de la vida cristiana. Barcelona, Espańa: Centre de Pastoral Litúrgica

McTaggart, J. M. E. (1908). “The Unreality of Time”, Mind 17, pp.457-47

Mellor, D.H. (1998). Real Time II, Londres, Inglaterra: Routledge

Pickstock, C. (1998). After Writing. On the Liturgical Consummation of Philosophy. Oxford, Inglaterra: Wiley-Blackwell

Pickup, M- (2015), "Real Presence in the Eucharist and Time-Travel”, Religious Studies 51, pp.379-389

Pitre, B. (2011), Jesus and the Jewish Roots of the Eucharist. Unlocking the Secrets of the Last Supper. New York, EE.UU.: Image 
Pruss, A. R. (2009), “The Eucharist: Real Presence and Real Absence” en Thomas P. Flint y Michael C. Rea (eds.), The Oxford Handbook of Philosophical Theology. Oxford, Inglatera: Oxford University Press, pp.512-537

Pruss, A. R. (2013), “Omnipresence, Multilocation, the Real Presence and Time Travel”, Journal of Analytic Theology 1 No 1, 60-73

Quine, O. (1973). Filosofía de la lógica, Madrid, España: Alianza

Rea, M.C. (2003). "Four-Dimensionalism". The Oxford Handbook of Metaphysics, Oxford, Inglaterra: Oxford University Press, pp. 246-280

Theodore Sider. (2001). Four-Dimensionalism. An Ontology of Persistence and Time. Oxford, Inglaterra: Clarendon Press

Smith, N. (2013). "Time Travel” Stanford Encyclopedia of Philosophy. Recuperado de: https://plato.stanford.edu/entries/time-travel/. Consultado el 7 de febrero de 2017.

Steward, H. (1997). The Ontology of Mind. Events, Processes, and States. Oxford, Iglaterra: Clarendon Press.

Santo Tomás de Aquino. (1952) Summa theologiae. Cura et studio Petri Caramello ex recensione leonina. Roma, Italia: Marietti. 